\title{
Strongly disordered spin ladders
}

\author{
R. Mélin ${ }^{1}, Y .-C$. Lin $^{2}$, P. Lajkó ${ }^{3}$, H. Rieger ${ }^{4}$, and F. Iglói ${ }^{1,3,5}$ \\ 1 Centre de Recherches sur les Trés Basses Températures* , B. P. 166, F-38042 Grenoble, France \\ 2 NIC, Forschungszentrum Jülich, 52425 Jülich, Germany \\ ${ }^{3}$ Institute for Theoretical Physics, Szeged University, H-6720 Szeged, Hungary \\ 4 Theoretische Physik, Universität des Saarlandes, 66041 Saarbrücken, Germany \\ ${ }^{5}$ Research Institute for Solid State Physics and Optics, H-1525 Budapest, P.O.Box 49, Hungary
}

(October 31, 2018)

\begin{abstract}
The effect of quenched disorder on the low-energy properties of various antiferromagnetic spin ladder models is studied by a numerical strong disorder renormalization group method and by density matrix renormalization. For strong enough disorder the originally gapped phases with finite topological or dimer order become gapless. In these quantum Griffiths phases the scaling of the energy, as well as the singularities in the dynamical quantities are characterized by a finite dynamical exponent, z, which varies with the strength of disorder. At the phase boundaries, separating topologically distinct Griffiths phases the singular behavior of the disordered ladders is generally controlled by an infinite randomness fixed point.
\end{abstract}

\section{INTRODUCTION}

Low dimensional quantum spin systems, chains and ladders are fascinating objects, which are the subject of intensive experimental and theoretical research. The main source of this activity is due to the observation that quantum fluctuations could result in qualitatively different low-energy behavior in these interacting many-body systems. It was first Haldanel who conjectured that antiferromagnetic (AF) spin chains with integer spin have a gap in the energy spectrum (Haldane phase), whereas the spectrum of chains with half-integer spins is gapless. By now a large amount of experimental and theoretical evidence have been collected in favor of the Haldane conjecture. It has been realized by Affleck, Kennedy, Lieb and Tasakil (AKLT) that the ground state structure of the Haldane phase for $S=1$ is closely related to that of the valence-bond solid model, where the ground state is built up from nearest-neighbor valence bonds. The hidden, topological order in the chain is measured by the non-local string order parameter 3 :

$$
O^{\alpha}=-\lim _{|i-j| \rightarrow \infty}\left\langle S_{i}^{\alpha} \exp \left(i \pi \sum_{l=i+1}^{j-1} S_{l}^{\alpha}\right) S_{j}^{\alpha}\right\rangle,
$$

where $S_{i}^{\alpha}$ is a spin- 1 operator at site $i, \alpha=x, y, z$ and $\langle\ldots\rangle$ denotes the ground state expectation value.

Another source of activity in the field of lowdimensional quantum spin strstems is due to the discovery of spin ladder materials spin ladders with even number of legs have a gapped spectrum, whereas the spectrum of odd-leg ladders is gapless 5 . For two-leg ladders, which are analogous objects to $S=1$ spin chains, the ground state structure can be related to nearest-neighbor valence bonds and a topological hidden order parameter, similar to that in Eq.(1.1) can be defined6.

More recently, ladder models with competing interactions, such as with stagogered dimerization and with rung and diagonal couplings 6 , have been introduced and studied. In these models, depending on the relative strength of the couplings, there are several gapped phases with different topological order, which are separated by firstor second-order phase transition lines.

Disorder turns out to play a crucial role in some experiments on low dimensional magnets. For instance, the NMP-TCNQ compound 8 can be well described by $S=1 / 2$ spin chains with random AF couplings. More recently, non magnetic sybsfitutions in low dimensional oxydes such as $\mathrm{CuGeO}_{3} 12$ (being a spin-Peierls compound), $\mathrm{PbNi}_{2} \mathrm{~V}_{2} \mathrm{P}_{8} \mathrm{13}$ (being a Haldane gap compound) or $\mathrm{Y}_{2} \mathrm{BaNiO}_{5} 14-19$ (being a Haldane gap compound) have been the subject of intense investigations. The essential feature of these compounds is the appearance of antiferromagnetism at low temperature which can be well described by the effective low energy models introduced in Refs. 19 21. $\mathrm{Sr}\left(\mathrm{Cu}_{1-x} \mathrm{Zn}_{x}\right)_{2} \mathrm{O}_{3}$ is a realization of the two-leg ladder, and can be doped by $\mathrm{Zn}$, a non magnetic ion23. The specific heat and spin susceptibility experiments indicate that the doped system is gapless even with low doping concentrations. We note that the experimentally found phase diagram of this compound, as well as other quantities, such as staggered susceptibility he been obtained by quantum Monte Carlo simulations 24.

*U.P.R. 5001 du CNRS, Laboratoire conventionné avec l'Université Joseph Fourier 
Theoretically, spin chains in the presence of strong disorder can be conveniently studied by a real-space renormalization group (RG) method introduced by Ma, Dasgupta and $\mathrm{Hu} 25(\mathrm{MDH})$. In this method strong bonds in the system are successively eliminated and other bonds are replaced by weaker ones through a second order perturbation calculation. As realized later by Fisher 26 for the random spin- $1 / 2$ chain and for the related model of random transverse-field Ising spin chain 27 the probability distribution of the couplings under renormalization becomes broader and broader without limit and therefore the system scales into an infinite randomness fixed point (IRFP), where the MDH renormalization becomes asymptotically exact. Fisher has also succeeded to solve the fixed-point RG equations in analytical form and to show, that for any type of (non-extremely singular) initial disorder the system scales inte the same IRFP. Later numerical28 30 and analytical 30 work has confirmed Fisher's results.

Generalization of the MDH approach for AF chains with larger values of the spin is not straightforward, since for not too strong initial disorder the generated new couplings could exceed the value of the already decimated ones. To hand this problem for the $S=1$ chain Hyman and Yano 31 and independently Monthus, Golinelli and Jolicaeur 32 have introduced an effective model with spin- 1 and spin- $1 / 2$ degrees of freedom and with random $\mathrm{AF}$ and ferromagnetic $(\mathrm{F})$ couplings. From an analysis of the RG equations they arrived to the conclusion that the IRFP of the model will be attractive if the original distribution parametrized by the power-law form

$$
P_{\text {pow }}(J)=\frac{1}{D} J^{-1+1 / D} \text {. }
$$

is strongly random, i.e. if $0<D^{-1}<D_{1}^{-1}$. For weaker initial disorder the system is still gapless, which is called the gapless Haldane phase.

Theoretical work about disordered spin ladders is mainly concentrated on the weak disorder limit. Results in this direction are obtained in the weak interchain coupling limit via the bosonization apprach 33 and by the random mass Dirac fermion method 34 . In particular a remarkable stability of the phases of the pure system against disorder with $X Y$ symmetry has been observed 33 .

In the experimental situation, however, as described before the effect of disorder is usually strong and we are going to consider this limiting case in this paper. Our aim is to provide a general theoretical background for strongly disordered spin ladders by studying in detail several models (conventional ladder, dimerized ladder, zig-zag ladder, and the full ladder with rung and diagonal couplings), which could have experimental relevance. Since often a small change in the couplings or in the strength of disorder could cause large differences in the low-energy singular properties of the models, therefore we have studied the phase diagrams in the space of several parameters. As a method of calculation we used a numerical implementation of the $\mathrm{MDH}$ approach, which could treat the combined effect of disorder, frustration, correlations and quantum fluctuations, while some problems are also studied by density matrix renormalization (DMRG). In particular we have investigated the stability of the different topologically ordered phases and studied the region of attraction of the IRFP.

The structure of the paper is the following. In Sec. 2 we define different spin ladder models and present their phase diagram for non-random couplings. A short overview about the MDH RG method and its application to random spin chains are given in Sec. 3. Our results about random spin ladders are presented in Sec. 4 and discussed in Sec. 5 .

\section{THE MODELS AND THEIR PHASE DIAGRAM FOR NON-RANDOM COUPLINGS}

We start with two spin-1/2 Heisenberg chains, labeled by $\tau=1,2$ and described by the Hamiltonians

$$
H_{\tau}=\sum_{l=1}^{L} J_{l, \tau} \mathbf{S}_{l, \tau} \mathbf{S}_{l+1, \tau}
$$

where $\mathbf{S}_{l, \tau}$ is a spin-1/2 operator at site $l$ and on chain $\tau$ and $J_{l, \tau}>0$. For non-random spin chains dimerization can be introduced as

$$
J_{l, \tau}=J\left[1+\gamma(-1)^{l+n(\tau)}\right], \quad 0 \leq \gamma<1,
$$

with $n(\tau)=0,1$, whereas for random dimerized couplings the even and odd bonds are taken from different distribution. The pure chain without dimerization $(\gamma=0)$ has a gapless spectrum, and spin-spin correlations decay as a power for large distance, which is called as quasi-longrange-order (QLRO). Introducing dimerization for $\gamma>0$ a gap opens in the spectrum 35 , which is accompanied by non-vanishing dimer order, $O_{\text {dim }}^{\alpha} \neq 0$. This is measured as the difference between the string order parameters in $\mathrm{Eq}(1.1)$ calculated with spin-1/2 moments at even (e) and odd (o) sites:

$$
O_{d i m}^{\alpha}=O_{e}^{\alpha}-O_{o}^{\alpha}
$$

In the following we generally consider non-dimerized chains, otherwise it is explicitly mentioned.

Now we introduce the interchain interaction

$$
H_{R}=\sum_{l=1}^{L} J_{l}^{R} \mathbf{S}_{l, 1} \mathbf{S}_{l, 2},
$$

which describes the usual rung coupling between the ladders (see Fig. 1.a). The conventional ladder model is described by the Hamiltonian: $H=H_{1}+H_{2}+H_{R}$. In the pure model by switching on the AF rung couplings, $J_{l}^{R}=J^{R}>0$, a Haldane-type gap opens above the ground state and the system has a non-vanishing even string topological order, which is measured by 6.36 : 


$$
O_{\text {even }}^{\alpha}=-\lim _{|i-j| \rightarrow \infty}\left\langle\left(S_{i+1,1}^{\alpha}+S_{i, 2}^{\alpha}\right) \exp \left(i \pi \sum_{l=i+1}^{j-1}\left(S_{l+1,1}^{\alpha}+S_{l, 2}^{\alpha}\right)\right)\left(S_{j+1,1}^{\alpha}+S_{j, 2}^{\alpha}\right)\right\rangle .
$$

For strong AF rung couplings every spin-pair on the same rung form a singlet, therefore this phase is called the rung singlet (RUS) phase.

(a)

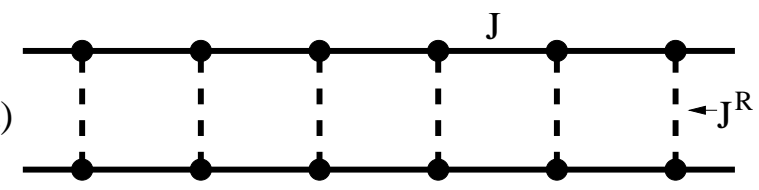

(b)

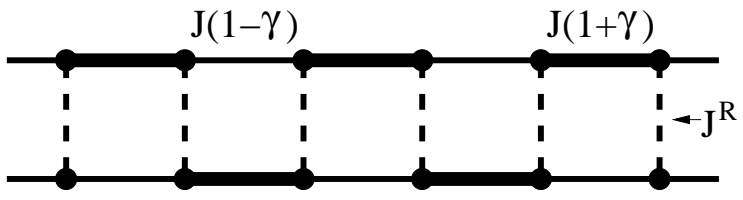

(c)



(d)



(e)

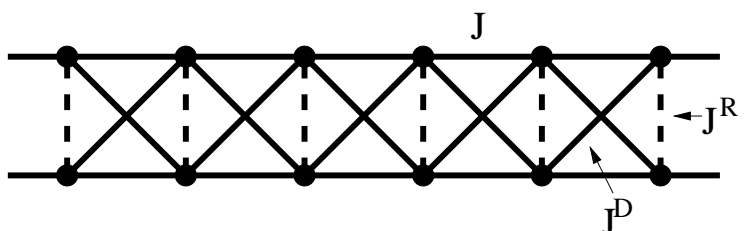

FIG. 1. Spin-ladder models used in the paper. The conventional two-leg ladder (a) and staggered dimerization in the chain couplings (b). The zig-zag ladder (c) and its representation as a chain with first and second neighbor couplings (d). The full ladder with rung and diagonal couplings (e).

Dimerization of the chain couplings could occur in two different ways. For parallel dimerization, when equal bonds in the two chains are on the same position, i.e. in Eq.(2.2) $n(1)=n(2)$, the combined effect of rung coupling and dimerization will always result in a gapped phase. In the other possible case of staggered dimerization, i.e. with $n(1)=-n(2)$ (see Fig. 1. 1.b), the two chains have an opposite dimer order, which competes with the rung coupling. As a result the phase diagram of the system (see Fig. 2) consists of two gapped phases, which are separated by a gapless transition line, starting in the pure, decoupled chains limit

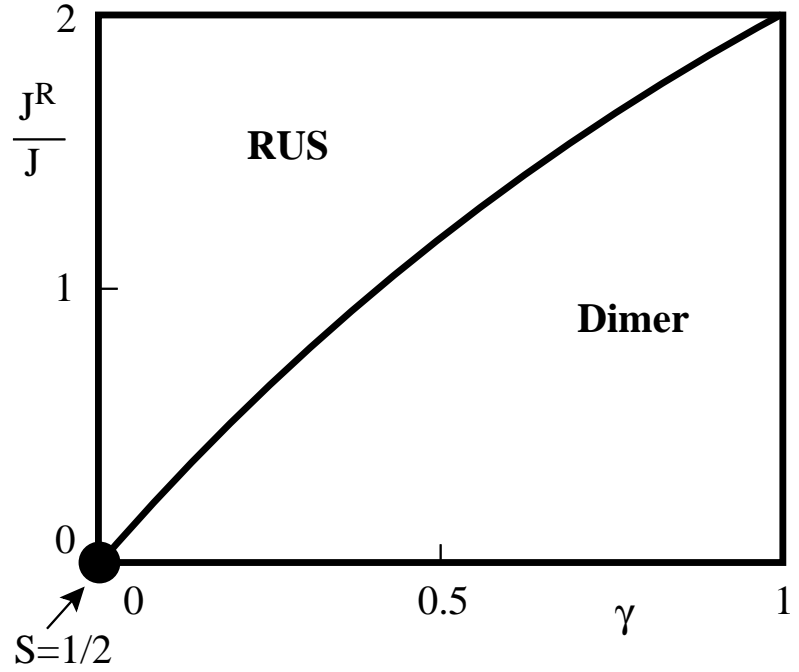

FIG. 2. Schematic phase diagram of the two-leg AF ladder with staggered dimerization (see Fig. 1.b for the definition of the couplings.) At the phase boundary between the rung singlet and dimer phases the gap vanishes.

Next, we extend our model by diagonal interchain couplings, given by the Hamiltonian term:

$$
H_{Z}=\sum_{l=1}^{L} J_{l}^{Z} \mathbf{S}_{l, 2} \mathbf{S}_{l+1,1}
$$

The complete Hamiltonian, $H=H_{1}+H_{2}+H_{R}+H_{Z}$, describes a zig-zag ladder (see Fig. 11.c) or can be considered as a spin chain with nearest neighbor $\left(J_{l}^{R}, J_{l}^{Z}\right)$ and next-nearest neighbor $\left(J_{l}\right)$ couplings (Fig. 1. d). The pure model with $J_{l}^{R}=J_{l}^{Z}=J_{1}$ and $J_{l}=J_{2}$ has two phases: a gapless phase for $J_{2} / J_{1}<.24$ is separated from a gapped phase by a quantum phase transition point.

Finally, we extend our model by two types of diagonal couplings, which are represented by the Hamiltonian:

$$
H_{D}=\sum_{l=1}^{L} J_{l}^{D}\left(\mathbf{S}_{l, 1} \mathbf{S}_{l+1,2}+\mathbf{S}_{l, 2} \mathbf{S}_{l+1,1}\right) .
$$

It is known that the pure AF diagonal ladder described by the Hamiltonian, $H=H_{1}+H_{2}+H_{D}$ with $J_{l}^{D}=J^{D}>0$ has a gapped spectrumb. Its ground state is of the AKLTtype and has a non-vanishing odd string order 36 , defined in analogy to Eq.(2.5)

$$
O_{\text {odd }}^{\alpha}=-\lim _{|i-j| \rightarrow \infty}\left\langle\left(S_{i, 1}^{\alpha}+S_{i, 2}^{\alpha}\right) \exp \left(i \pi \sum_{l=i+1}^{j-1}\left(S_{l, 1}^{\alpha}+S_{l, 2}^{\alpha}\right)\right)\left(S_{j, 1}^{\alpha}+S_{j, 2}^{\alpha}\right)\right\rangle .
$$


In the full ladder there are both rung and diagonal couplings (see Fig. 1.e) and it is described by the Hamiltonian $H=H_{1}+H_{2}+H_{R}+H_{D}$. For non-random $\mathrm{AF}$ couplings there is a competition between rung and diagonal couplings, so that the ground state phase diagram of the system consists of two topologically distinct gapped phases (see Fig. 3). The phase transition between the two phases is of first order 6 .

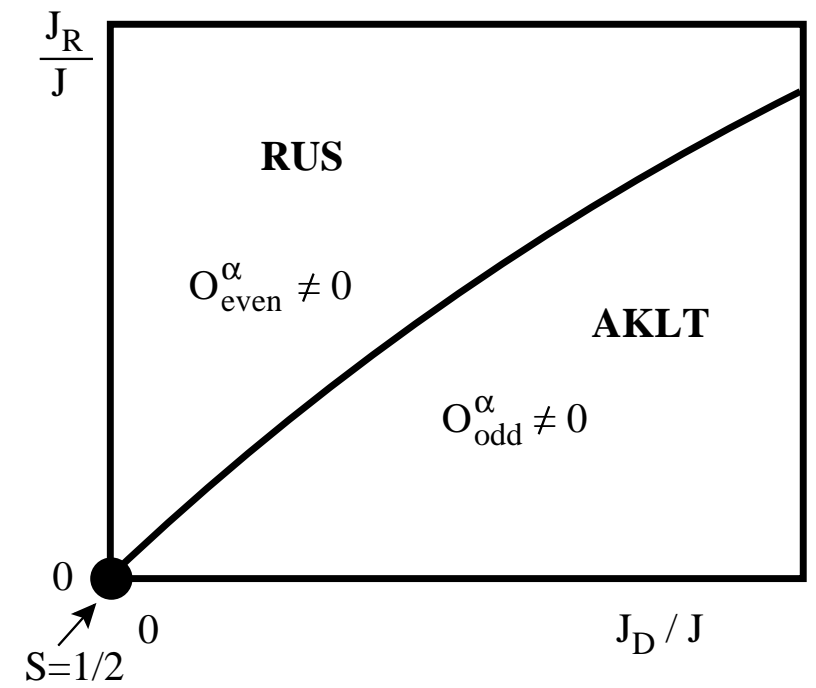

FIG. 3. Schematic phase diagram of the full AF ladder with homogeneous rung and diagonal couplings. The transition between the two topologically distinct gapped phases is of first order, except of the limit $J_{R}=J_{D}=0$.

The main subject of our paper is to investigate how the phase diagrams of the pure ladder models, in particular in Figs. 2 and 3 are modified due to the presence of quenched disorder.

\section{THE MDH RENORMALIZATION: RESULTS FOR SPIN CHAINS}

In the $\mathrm{MDH}$ renormalization group method for random spin-1/2 chains the random AF bonds are arranged in descending order according to their strength, and the strongest bond, say $J_{23}$, connecting sites 2 and 3 sets the energy scale in the problem, $\Omega=J_{23}$. We denote by 1 the nearest neighbor site to 2 with a connecting bond $J_{12}$ and similarly denote by 4 the nearest neighbor site to 3 with a connecting bond $J_{43}$. If $J_{23}$ is much larger then the connecting bonds the spin pair $(2,3)$ acts as an effective singlet. It follows that the strongly correlated singlet pair can be frozen out. Due to the virtual triplet exciations, an effective coupling $\tilde{J}_{14}$ is generated between the sites 1 and 4 . These two sites become nearest neighbors once the singlet has been eliminated. In a second-order perturbation calculation one obtains

$$
\tilde{J}_{14}=\kappa \frac{J_{12} J_{43}}{\Omega}, \quad \kappa(S=1 / 2)=1 / 2 .
$$

The new coupling is thus smaller than any of the original ones. The energy scale $\Omega$ is continuously reduced upon iterating the procedure and at the same time the probability distribution of the couplings $P(J, \Omega)$ approaches a limiting function. In a gapless random system $\Omega$ tends to zero at the fixed point of the transformation and the low energy tail of the distribution is typically given by:

$$
P(J, \Omega) \mathrm{d} J \simeq \frac{1}{z}\left(\frac{J}{\Omega}\right)^{-1+1 / z} \frac{\mathrm{d} J}{\Omega} .
$$

The dynamical exponent $z$ determines how the length scale $L$ scales with the time scale $\tau$ :

$$
\tau \sim \Omega^{-1} \sim L^{z}
$$

In general $z$ is not a universal quantity: its value depends on the form of the original disprder. However $z$ stays invariant under renormalization 37 . Therefore one can deduce its value from the renormalized distribution in Eq. 3.2). Varying the parameters of the initial distribution one can reach a situation where the width of the distribution in Eq.(3.2) grows without limits, i.e. $z$ formally tends to infinity. In this case, according to exact results on the random $\mathrm{AF}$ spin- $1 / 2$ chain 26 , one should formally replace $z$ in Eq.(3.2) by $-\ln \Omega$, so that the scaling relation in Eq. 3.3) takes the form:

$$
\ln t_{r} \sim L^{\psi}, \quad \psi=1 / 2
$$

This type of fixed point, where the ratio of any two neighboring bonds typically tends to zero or infinity, is called an infinite randomness fixed point (IRFP). It has been conjectured that the $\mathrm{MDH}$ renormalization group transformation (3.1) leads to exact results regarding the singular properties of the transformation, namely the value of $\psi$ in Eq. (3.4) is exact 26.42 .

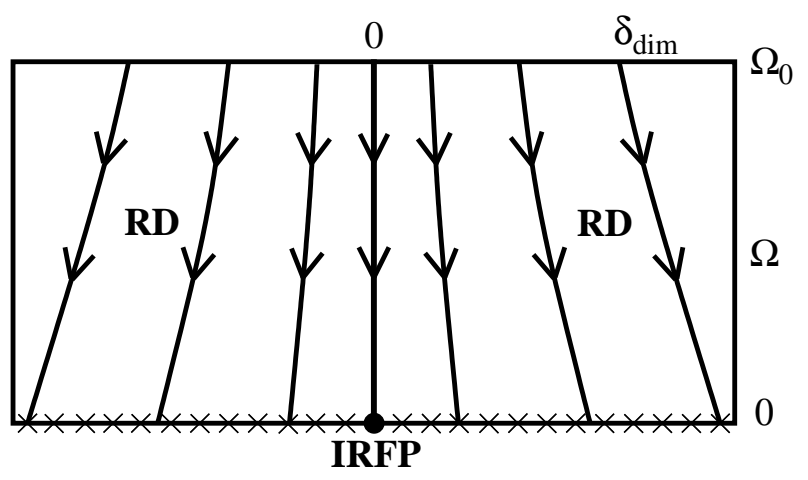

FIG. 4. Schematic RG phase diagram of the random dimerized $s=1 / 2$ chain as a function of the quantum control parameter $\delta_{\text {dim }}$ and the energy scale $\Omega$. Along the RG trajectories the dynamical exponent $z\left(\delta_{\text {dim }}\right)$ is constant. The non-dimerized model with $\delta_{\text {dim }}=0$ is attracted by the IRFP with $1 / z=0$. With decreasing the energy scale $\Omega$, disorder in the system is increasing. 
For the random $\mathrm{AF}$ spin- $1 / 2$ chain, according to exact results 26 any amount of disorder is sufficient to drive the system into the IRFP. Similarly, the gapped dimer phase will turn into a gapless random dimer phase for any amount of disorder, where the dimerization parameter is defined as

$$
\delta_{d i m}=\left[\ln J_{o d d}\right]_{\mathrm{av}}-\left[\ln J_{\text {even }}\right]_{\mathrm{av}}
$$

in terms of the couplings $J_{\text {odd }}$ and $J_{\text {even }}$ at odd and even sites, respectively. The random dimer phase is a quantum version of the Griffiths-phase, which has been originally introduced for classical disordered systems 38 . The schematic RG-flow diagram of the random dimerized chain is drawn in Fig. 4 .

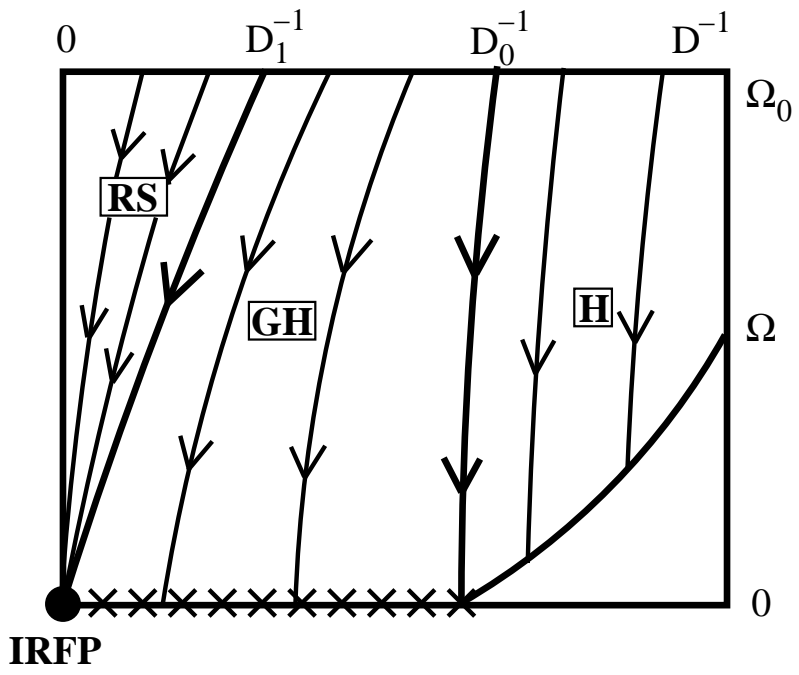

FIG. 5. Schematic RG phase diagram of the random AF spin-1 chain, as a function of the disorder strength $D$ and the energy scale $\Omega$. For weak disorder, $D<D_{0}$, there is a Haldane ("H") gap in the spectrum. For intermediated disorder, $D_{0}<D<D_{1}$ the system is in the gapless Haldane ("GH") phase with a varying dynamical exponent $z(D)$. For strong enough disorder, $D>D_{1}$ the system is in the random singlet phase and scales into the IRFP.

The MDH method has also been used to study the singular properties of the random AF spin- 1 chain. Here we first note that the spectrum of the pure system has a Haldane gap, which is stable against weak randomness. Consequently the MDH renormalization, which is by definition a strong disorder approach, becomes valid if the initial disorder is increased over a limit, say $D_{0}$. Our second remark concerns the "projecting onto the lowest level" procedure 39 when after decimating out a strongly coupled singlet the generated new coupling is in the form of Eq.(3.1), however with a constant of $\kappa(S=1)=4 / 3$. Consequently at the initial RG steps the energy scale could behave non-monotonically, so that an IRFP behavior is expected only for strong enough initial disorder. To this problem a modified RG scheme was proposed 31. 22, which is based on the principle of "pro- jecting out the highest level". By this method an effective Hamiltonian with spin- 1 and spin- $1 / 2$ degrees of freedom has been introduced, where between the spins both $\mathrm{AF}$ and $\mathrm{F}$ couplings could be present but their distribution should respect some constraints. The RG analysis of this effective model leads to two different types of strongly disordered phases, provided the disorder of the original distribution exceeds the limiting value of $D_{0}$. For an intermediate range of disorder, so that $D_{0}<D<D_{1}$ the system scales into a quantum Griffiths-phase, the so-called gapless Haldane phase, where $z=z(D)$ is a monotonously increasing function of disorder and $1 / z(D)>0$. When the strength of disorder exceeds a second limit, say $D>D_{1}>D_{0}$, the dynamical exponent becomes infinite and the singular behavior of the system is controlled by the IRFP (see Fig 5).

Till now there are no numerical estimates about the limiting disorder strengths, $D_{0}$ and $D_{1}$. For the uniform distribution, which corresponds to $D=1$ in Eq. (1.2) the system is in the gapless Haldane phase with $z \approx 1.54$

Finally, we mention the work by Westerberg et al.41 about renormalization of spin- $1 / 2$ Heisenberg chains with mixed $\mathrm{F}$ and AF couplings. In this problem, due to the presence of strong $\mathrm{F}$ bonds under renormalization spin clusters with arbitrary large effective moment $S^{\text {eff }}$ are generated, such that $S^{e f f} \sim \Delta^{-\omega}$, where $\Delta$ is the largest local gap in the system and $\Delta \rightarrow 0$ at the fixed point. Singularities of different physical quantities are related to the scaling exponent $\omega$.

\section{RENORMALIZATION OF AF SPIN LADDERS}

With a ladder geometry, spins are more interconnected than in a chain, which leads to a modification of the decimation procedure described in the previous section. As shown in Fig. 6 both spins of a strongly coupled pair, say $(2,3)$, are generally connected to the nearest neighbor spins, denoted by 1 and 4 . After decimating out the singlet pair the new, effective coupling between 1 and 4 is of the form:

$$
\tilde{J}_{14}^{e f f}=\kappa \frac{\left(J_{12}-J_{13}\right)\left(J_{43}-J_{42}\right)}{\Omega}, \quad \kappa(S=1 / 2)=1 / 2,
$$

which should replace Eq.(3.1) obtained in the chain topology, i.e. with $J_{13}=J_{42}=0$. With the rule in Eq.(4.1) F couplings are also generated. As a consequence, the renormalized Hamiltonian contains both AF and $\mathrm{F}$ bonds. When at some step of the renormalization an $\mathrm{F}$ bond becomes the strongest one, it will lead to the formation of an effective spin-1 cluster. In further RG steps the system renormalizes into a set of effective spin clusters having different moments and connected by both $\mathrm{AF}$ and $\mathrm{F}$ bonds. The detailed renormalization rules have already been given in Ref.21. 


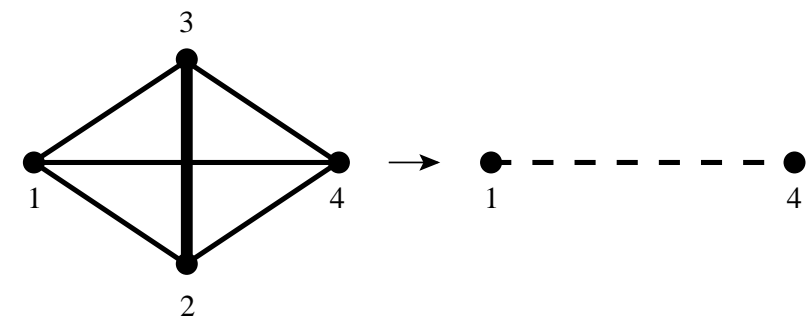

FIG. 6. Singlet formation and decimation in the ladder geometry.

Due to the ladder topology and the complicated renormalization rules the RG equations can not be treated analytically and one resorts to numerical implementations of the renormalization procedure. We note that a variant of the MDH renormalization has been successfully applied numerically for the two dimensional random transverse-field Ising model (RTIM) 2243 (also for double chains of the RTIM43 . An IRFP has been obtained both for the 2D RFIM 2233 and the double chain RFIM 43 .

In practice we use a finite size version of the $\mathrm{MDH}$ renormalization, as for the RTIM in Ref 43. In this method we start with a finite ladder of $L$ sites and with periodic boundary conditions and perform the decimation procedure until one spin pair with a first gap $\Delta$ remains in the system. Since $\Delta$ plays the role of the energy scale at length scale $L, \Delta$ and $L$ should be related by the relation (3.3) involving the dynamical exponent $z$. Performing the above decimation for different samples the probability distribution of $\Delta$ in the small $\Delta$ limit is described by the form in Eq.(3.2), where the energy scale $\Omega$ is replaced by $L^{-z}$.

The IRFP is signalled by a diverging $z$, or more precisely the $P_{L}(\Delta) \mathrm{d} \Delta$ distributions have strong $L$ dependence, so that the appropriate scaling combination is

$$
\ln \left(L^{\psi} P_{L}(\Delta)\right) \simeq f\left(L^{-\psi} \ln \Delta\right),
$$

which can be obtained from Eq.(3.2) by formally setting $z \simeq-\ln \Delta \sim L^{\psi}$.

In the actual calculations we have considered several 100.000 realizations of random ladders of length up to $L=512$. Then, from the distribution of the gap at the last step of the RG iteration we have calculated the dynamical exponent, $z$. The random couplings were taken from the power-law distribution in Eq.(1.2), where the strength of disorder is measured by the parameter $D$. In the following we present our results for the specific ladder models discussed in Sec. 2.

\section{A. Random conventional ladders}

We start with the conventional ladders in Fig. 1.a where the couplings along the chains $\left(J_{l}^{\tau}, \tau=1,2\right)$ and the couplings along the rungs $\left(J_{l}^{R}\right)$ are taken from the same random distributions. In Fig. 目 we show the probability distribution of the gaps at the last step of the RG iteration calculated with the disorder parameter $D=1$ (see Eq.(1.2)). As seen in the figure the small energy tail of the distribution follows the functional form given by Eq. (3.2) and the dynamical exponent $z$ given by the asymptotic slope of the distributions is finite and has only a very weak size dependence.

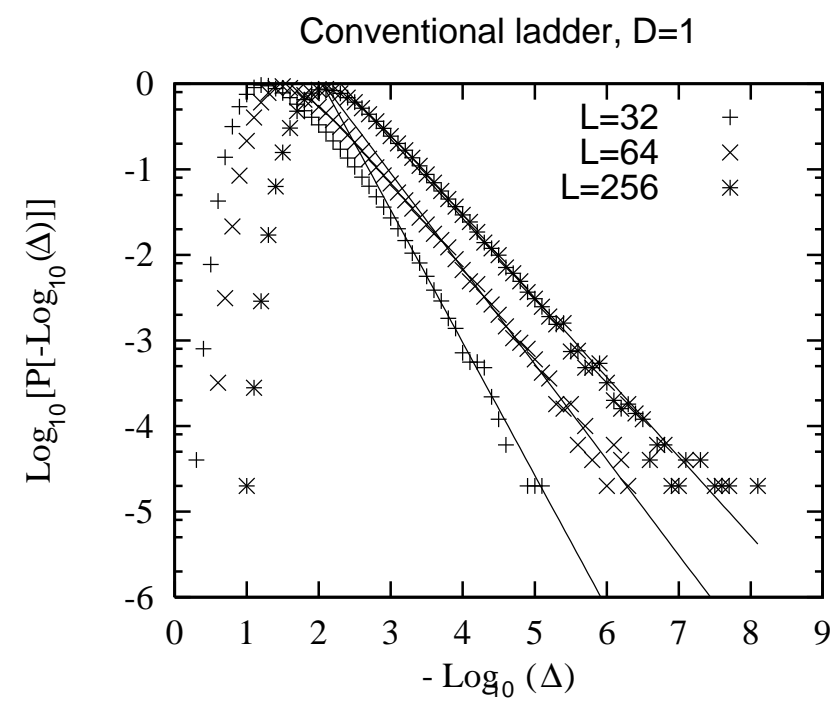

FIG. 7. Probability distribution of the first gaps for the conventional random ladder with a disorder $D=1$ (see Eq.(1.2) and system size $L=32, L=64$, and $L=256$. For clarity, we have not shown the data corresponding to $L=128$. The solid lines represent the best fit to the form $\log _{10}\left[P\left[-\log _{10} \Delta\right]\right]=A_{L}-\frac{1}{z_{L}} \log _{10} \Delta$, with $A_{32}=3.18$, $A_{64}=2.30, A_{128}=1.92, A_{256}=2.14$ and $z_{32}=0.65$, $z_{64}=0.90, z_{128}=1.06$, and $z_{256}=1.07$. We deduce that the asymptotic value of the dynamical exponent is $z_{\infty} \simeq 1.07$.

Repeating the calculation for other values of $D$ we obtain a set of $D$-dependent dynamical exponents which are represented on Fig. 8. For strong disorder we obtain $z(D)<D$, which means that disorder is reduced in the course of the renormalization. In the terminology of Motrunich et al 42, this system is a finite randomness system, as opposed to the infinite randomness systems that will be considered in the next subsections. For weak disorder the dynamical exponent predicted by the approximative $\mathrm{MDH}$ renormalization is lowered below one for $D<D_{0} \approx 1$. Here we argue that in this region the effect of disorder is irrelevant, so that the system is in the gapped RUS phase. Indeed, in a pure quantum system, where scaling in time and space is isotropic, the dynamical exponent is $z_{\text {pure }}=1$. Similarly, for disorder induced gapless systems, where disorder in the time direction is strictly correlated, the dynamical exponent can not be smaller, than in the pure system, so that $z_{\text {dis }} \geq z_{\text {pure }}=1$. Consequently, if the disorder induced dynamical exponent is $z_{\text {dis }}<1$, then disorder could only influence the correction to scaling behavior, but the system stays gapped. In view of this remark $D_{0}$ can be 
considered as the lower limiting value of the disorder, where the conventional finite randomness behavior ends. So that the phase diagram of random conventional twoleg spin ladders consists of two phases: a gapped RUS (Haldane) phase and a random gapless Haldane phase. The latter is characterized by a finite dynamical exponent $z(D)$ for any strong but finite initial disorder. Consequently there is an important difference with the random AF spin-1 chain which flows into the IRFP above a finite critical value of randomness (see Fig. 5).

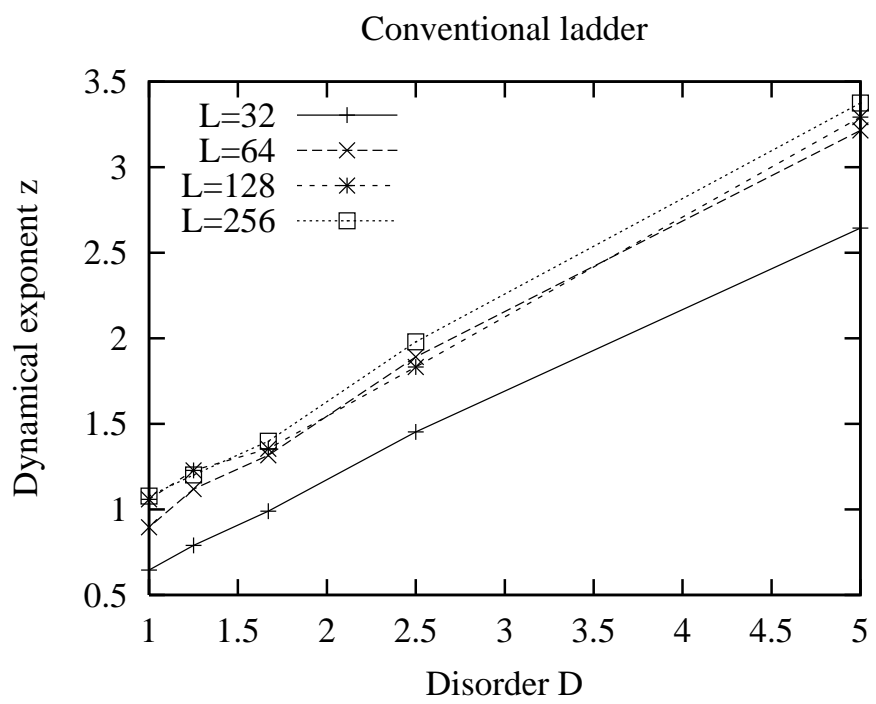

FIG. 8. Variation of dynamical exponent $z$ versus disorder $D$ for the conventional ladder with lengths $L=32, L=64$, $L=128$, and $L=256$. For large system sizes and strong disorder, one has $z_{\infty} \simeq 0.42+0.58 D<D$. In the region with $z_{\infty}<1$, where the disorder is irrelevant, the system is in the gapped Haldane phase (see text).

\section{B. Random ladders with staggered dimerization}

In this subsection we consider conventional ladders with staggered dimerization having a dimerization parameter, $0 \leq \gamma \leq 1$, in Eq.(2.2). The different type of couplings in the ladder are taken from the power-law distribution in Eq.(1.2), each having the same disorder parameter, $D$, however the range of the distribution for the different type of couplings are, $0<J_{l}^{R}<J_{\max }^{R}$ for the rung couplings, $0<J_{l}^{\text {weak }}<(1-\gamma) J_{\max }$ and $0<J_{l}^{\text {strong }}<(1+\gamma) J_{\max }$ for the weaker and stronger chain couplings, respectively. For a fixed value of $\gamma$ and $D$ we have calculated the finite-size dependent effective dynamical exponent, $z$, as a function of the coupling ratio, $J_{\max }^{R} / J_{\max }$.

As shown in Fig. 9 the effective exponents have the same type of qualitative behavior for different values of the dimerization parameter, $\gamma$. In each case the curves have a maximum at some value of the couplings, where the finite-size dependence is the strongest, whereas more far from the maximum the convergence of the data is faster. To decide about the possible limiting value of $z$, in particular at the maximum of the curves, we analyze the behavior for $\gamma=1$ in Fig. 9c, which is just a dimerized random chain, the properties of which are exactly known by some extent 26.30.


FIG. 9. Finite-size estimates of the dynamical exponent of random conventional ladders with staggered dimerization as a function of the coupling ratio, $J_{\max }^{R} / J_{\max }$, with a disorder parameter $D=1$ and for different dimerizations: a) $\gamma=0.5$, b) $\gamma=0.75$, c) $\gamma=1$.. In d) a similar calculation for the random $X X$-chain is presented $(D=1, \gamma=1)$, where the exact dynamical exponent in Eq. (4.3), obtained in the $L \rightarrow \infty$ limit, is shown by dashed line.

The random critical point of this system is situated at $J_{\max }^{R} / J_{\max }=2$, where the critical behavior is governed by an IRFP, so that the dynamical exponent, $z$, is formally infinity. For any other values of the couplings, $J_{\max }^{R} / J_{\max }$, the system is in the random dimer phase, where the dynamical exponent is finite and coupling dependent. To see the general tendency of finite-size convergence of the $z$ exponent around the critical point we have repeated the calculation at the $D=1, \gamma=1$ case for the random $X X$-chain and the numerical finite-size results are compared in Fig. 9d with the exact value of the dynamical exponent, as given by the solution of the equation:

$$
\frac{J_{\max }^{R}}{J_{\max }}=2\left(\frac{D^{2}}{D^{2}-z^{-2}}\right)^{-z}
$$

known from Ref. 37,30. As seen in Fig. 9 the dynamical exponents of random $X X$ - and Heisenberg-ladders have very similar coupling dependence and one expects the same type of divergence at the critical point for all values of $\gamma$. In Fig. 10 we illustrate the scaling behavior 
of the gap at the transition point, i.e. at the maximum of the curves in Fig. 9 a.

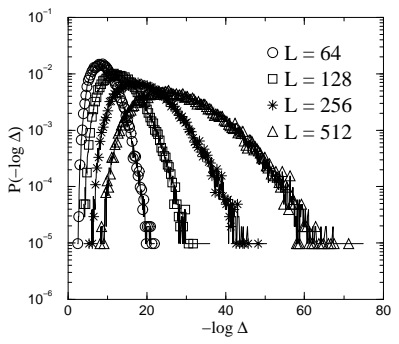

(a)

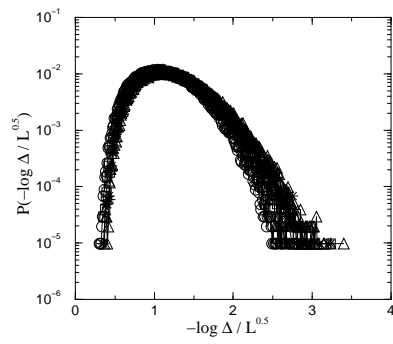

(b)
FIG. 10. a) Probability distribution of the first gap at the transition point of the random conventional ladder with staggered dimerization, $D=1, \gamma=0.5, J_{\max }^{R} / J_{\max }=1.1$. The distributions become broader and broader with $L$, which signals infinite randomness behavior. b) Scaling plot in terms of the scaling combination in Eq.(3.4).

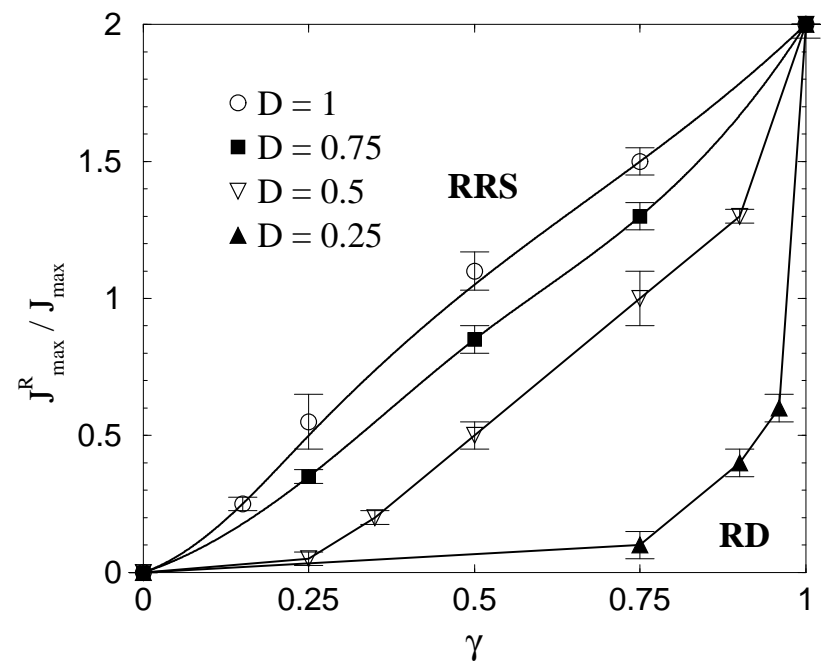

FIG. 11. Phase diagram of random conventional ladders with staggered dimerization for different disorder parameters. The random dimer (RD) phase and the random rung singlet (RRS) phase are separated by a random critical line of infinite randomness behavior.

The distributions in Fig. 10a become broder and broder with the size and the effective dynamical exponent is increasing with the size without limits. An appropriate scaling collaps of the gap-distributions has been obtained in Fig. 10 b, where the scaling variable in Eq.(1.2), with $\psi=1 / 2$ is used. Similar type of infinite randomness behavior is observed at other points of the critical lines, with the same exponent $\psi=1 / 2$, which turned out to be universal. Thus we conclude that the random conventional ladder with staggered dimerization has two Griffiths-type gapless phases, the random dimer phase and the random rung singlet phase, which are separated by a random critical line, along which there is infinite randomness behavior. For different disorder parameter, $D$, the position of the random critical line is modified, generally stronger disorder is in favor of the random rung singlet phase, see Fig. 11 .

We note that the previously studied random conventional ladder is contained as a special point in this phase diagram at $J_{\max }^{R} / J_{\max }=1$ and $\gamma=0$. This point is in the random rung singlet phase for any value of $D$, thus the dynamical exponent is finite in accordance with the previous results.

\section{Random zig-zag ladders}

For the zig-zag ladders the nearest neighbor couplings $\left(J_{l}^{R}=J_{l}^{Z} \equiv J_{l}^{1}\right)$ are taken from the power-law distribution in Eq.(1.2) with the coupling $J_{l}^{1}$ within the range $0<J_{l}^{1}<J_{\max }^{1}$. Similarly, the next-nearest neighbor couplings $\left(J_{l} \equiv J_{l}^{2}\right)$ are taken from the same type of power-law distribution and the range of couplings is now $0<J_{l}^{2}<J_{\max }^{2}$.

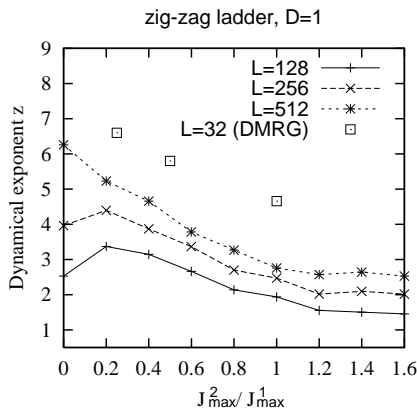

(a)

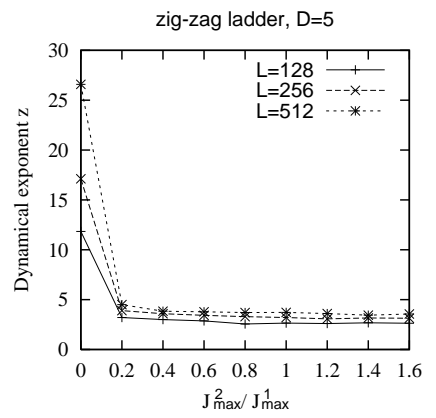

(b)
FIG. 12. Variation of the dynamical exponent $z$ versus $J_{\max }^{2} / J_{\max }^{1}$ for the zig-zag ladder with a disorder $D=1$ (a) and $D=5$ (b). For $D=1$, the $\mathrm{MDH}$ renormalization group data with $L=128, L=256$ and $L=512$ have been compared to the DMRG calculation with $L=32$ (see Fig. 13). The lines connecting the calculated points are guides to the eyes.

The calculated dynamical exponent, $z$, as shown in Figs. 12 has its maximum at $J_{\max }^{2} / J_{\max }^{1}=0$ and around this point one can observe strong finite-size dependence, the range of which is wide, in particular for weak disorder (see Fig. 12). At $J_{\max }^{2} / J_{\max }^{1}=0$, where the zig-zag ladder reduces to a random $\mathrm{AF}$ chain, the system is in the IRFP, thus the extrapolated value of the dynamical exponent is formally infinity. Given the strong finite-size corrections in the numerical RG data of the dynamical exponent 4 in Fig. 12, it is difficult to decide whether the IRFP behavior of the zig-zag ladders is extended to a finite region of the couplings $J_{\max }^{2} / J_{\max }^{1}>0$ or whether this region shrinks to a single point only. The first scenario may be related to the existence of a gapless phase of the pure model for $J^{2} / J^{1}<0.24$. 


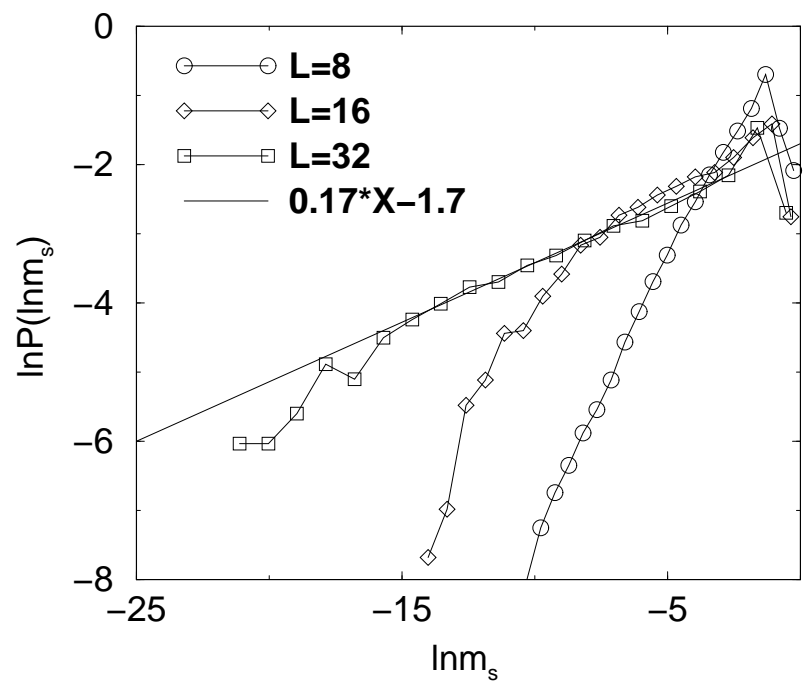

FIG. 13. Distribution of the log surface magnetization of the random zig-zag ladder using a power-law distribution $\left(D=1, J_{\max }^{2} / J_{\max }^{1}=0.5\right)$, for different lengths of the ladder, $L$. The asymptotic slope of the distribution, indicated by the straight line, is the inverse of the dynamical exponent, see Eq.(4.4).

To discuss this issue we have calculated the dynamical exponent by an independent method based on density matrix renormalization. In principle, the dynamical exponent is related to the distribution of the first gap, $\Delta$, in the small $\Delta$ limit, see in Eq.(3.2) with $\Delta \rightarrow J$. However, a precise numerical calculation of a small $\Delta$ by the DMRG method is very difficult, therefore we used another strategy, as described in details in Refs. 37,44. By this method one considers the equivalent $\mathrm{AF}$ chain with random first- and second-neighbor couplings (see Fig. 1d) and with fixed-free boundary conditions and calculate the surface magnetization, $m_{s}$, at the free end, which can be done very accurately by the DMRG method. As argued in Refs. 37, 44 for a random chain $m_{s}$ and $\Delta$ can be considered as dual quantities, so that the distribution of the surface magnetization is asymptotically given by

$$
P\left(\ln m_{s}\right) \sim m_{s}^{1 / z}, \quad m_{s} \rightarrow 0 .
$$

Thus the dynamical exponent $z$ can be obtained from an analysis of the small $m_{s}$ tail of the distribution, as illustrated in Fig. 13 where the distribution function of $\ln m_{s}$ is given in a log-log plot for different lengths of the ladder. As seen in this Figure the slope of the distribution is well defined for larger systems, from which one can obtain an accurate estimate for the dynamical exponent, which is finite. Repeating the calculation for other values of the coupling ratio, $J_{\max }^{2} / J_{\max }^{1}$, we have obtained a set of the dynamical exponents, which are plotted in Fig. 12. These accurate DMRG data show that the extrapolated values of the effective exponents calculated by the numerical RG-method are finite for any $J_{\max }^{2} / J_{\max }^{1}>0$. Consequently the random zig-zag ladder has just one IRFP at $J_{\max }^{2} / J_{\max }^{1}=0$, whereas the system in the region of $J_{\max }^{2} / J_{\max }^{1}>0$ is in a gapless random dimer phase. In view of the numerical results in Fig. 12, where $z_{\infty}$ seems to stay over $z_{\text {pure }}=1$, it is quite probable that the randum dimer phase exists for any small value of the disorder.

\section{Random $J_{1}-J_{2}$ ladders}

The full ladder, as represented in Fig. 1.e has three different type of couplings: $J_{l}, J_{l}^{R}$ and $J_{l}^{D}$. Here we consider a special case of this model, when the chain $\left(J_{l}\right)$ and rung $\left(J_{l}^{R}\right)$ couplings are taken from the same power-law distribution with a disorder parameter $D$ and having a range of $0<J_{l}, J_{l}^{R}<J_{\max }^{1}$. On the other hand the diagonal couplings are taken from the same type of power-law distribution and are within the interval $0<J_{l}^{D}<J_{\max }^{2}$. This model, having first- and second-neighbor interactions, is called a $J_{1}-J_{2}$ ladder. We have calculated the finite-size dynamical exponents as a function of the coupling ratio $J_{\max }^{2} / J_{\max }^{1}$ for different strengths of disorder (see Fig. 14). These curves show similar qualitative behavior as those calculated for the random conventional ladders with staggered dimerization in Figs. 9, so that we can draw similar conclusions.

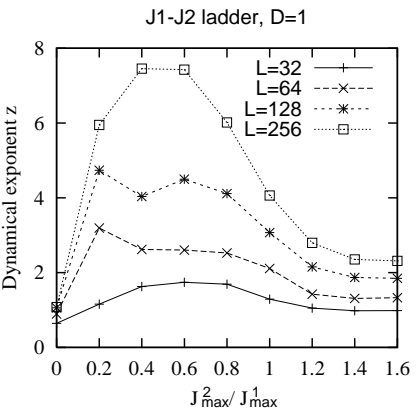

(a)

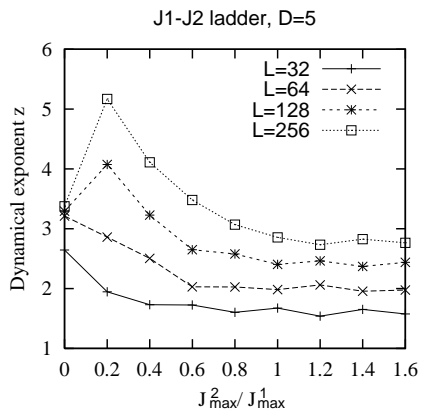

(b)
FIG. 14. Variation of the dynamical exponent $z$ versus $J_{\max }^{2} / J_{\max }^{1}$ for the random $J_{1}-J_{2}$ ladder with a disorder $D=1$ (a) and $D=5$ (b). The lines connecting the calculated points are guides to the eyes Note the strong finite-size corrections in the Griffiths phases 4 .

The extrapolated position of the maximum of the $z$ curves is identified as a quantum critical point with infinite randomness behavior. Indeed, repeating the calculation as indicated for the dimerized ladder model in Fig. 10 we obtained a scaling behavior as in Eq.(3.4). with an exponent which is compatible with $\psi=1 / 2$. The random quantum critical point separates two gapless Haldane phases, having odd and even topological order, respectively. 


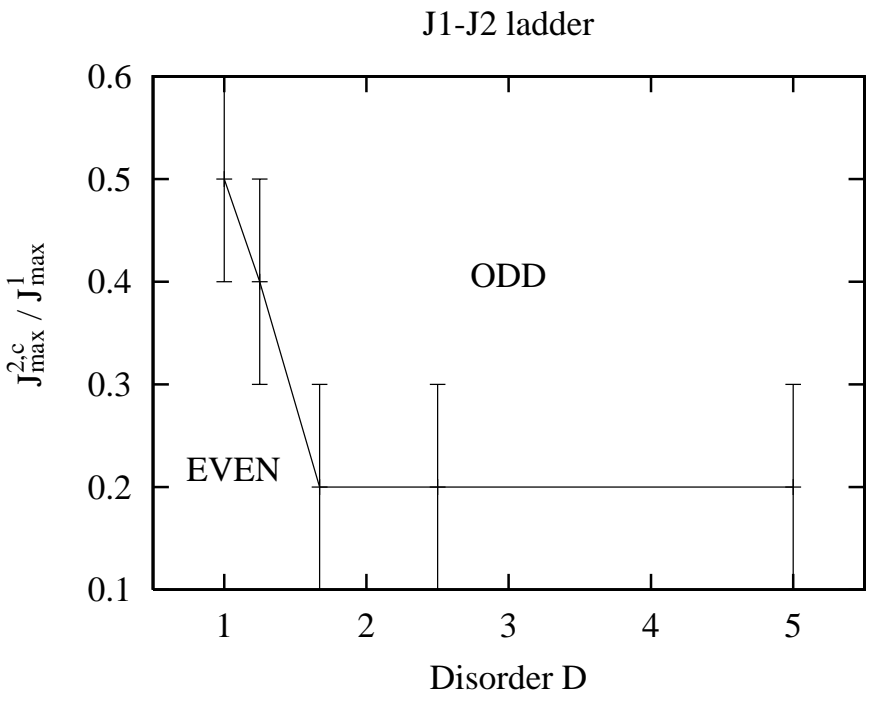

FIG. 15. Phase diagram of the random $J_{1}-J_{2}$ ladder obtained from the maximum in the variation of the dynamical exponent versus $J_{\max }^{2} / J_{\max }^{1}$ for the largest available sizes $L=256$ (see Fig. 14 for $D=1$ and $D=5$ ). The two straight lines connecting the calculated transition points are guides to the eyes. The whole transition line between the two phases with even and odd topological orders, respectively, is presumably a line of IRFPs.

Repeating the calculation for different disorder parameters we obtain a phase diagram shown in Fig. 15. In the range of disorder we used in the calculation the random critical point is always attracted by the IRFP, this property probably remains true for any small value of disorder.

\section{DISCUSSION}

In this paper different type of random AF spin ladder models have been studied by a numerical strong disorder RG method. In particular we asked the questions i) how the phase diagrams of the pure models are modified due to quenched disorder and ii) how the concepts observed in random $\mathrm{AF}$ chains, such as infinite randomness and Griffiths-type singularities are valid for these more complicated, quasi-one-dimensional models.

In our numerical calculations we observed as a general rule that for strong enough disorder the ladder models, like the random chains, become gapless. The dynamical exponent of the models is generally non-universal: $z$ depends on both the strength of disorder and on the value of the couplings. In models where there is a competition between different types of phases, either due to staggered dimerization or due to frustration, such as in the $J_{1}-J_{2}$ model, at the phase boundary the critical behavior of the random model is generally controlled by an infinite randomness fixed point, at least for strong enough disorder. The low-energy properties of the systems in this
IRFP are asymptotically exactly known from analytical calculations in random AF spin chainse2. 30. Thus the general phase diagram consists of Griffiths-type phases with different topological order separated by a random critical point of the IRFP type. The zig-zag ladder is an exception, where there is just one Griffiths phase and the random critical point is located at its boundary.

Next we turn to discuss about possible cross-over effects when the strength of disorder is varied. These problems can not be directly studied by the simple strong disorder RG method, however, from arguments considering the sign of $z_{\text {dis }}-z_{\text {pure }}$ and from analogous investigations on quantum spin chains 3132.44 we can suggest the following picture. Originally gapped phases could stay gapped for weak disorder and become gapless only if the strength of disorder exceed some finite limiting value, as seen for the random conventional ladder. However, for frustrated ladders, such as the zig-zag and the $J_{1}-J_{2}$ ladders, any small amount of disorder seems to bring the system into a random gapless phase. At a phase boundary, such as in the staggered dimerized ladder and the $J_{1}-J_{2}$ model, the random critical behavior is of the IRFP type, probably for any small amount of disorder.

At this point we comment on the similarity of the lowenergy behavior of spin chains with $2 S=$ odd $(2 S=$ even) spins and that of spin- $1 / 2$ ladders with $n=$ odd $(n=$ even $)$ legs. If the pure systems are gapless, i.e. $2 S=n=o d d$, strong enough disorder is expected to bring both systems into the IRFP. For $2 S=n=$ odd $\geq 3$, there is a limiting disorder strength, $D_{c}(n)$, below which the system is described by a conventional random fixed point with $z<\infty$. On the other hand for $2 S=n=$ even we have only a partial analogy: for weak disorder both systems are gapped, which turn into a gapless Griffithstype phase for stroger disorder. While the random ladder stays in this conventional random phase for any strength of the disorder the random spin chain will turn into a IRFP behavior at some finite limiting randomness. This type of infinite randomnass behavior can, however, be seen for frustrated even-leg $J_{1}-J_{2}$ ladders at the transition point. We can thus conclude that random ladders with even and odd number of legs belong to different universality classes.

Finally, we comment on random square lattice antiferromagnets, which can be obtained in the limit when the number of legs, $n$, goes to infinity. By increasing $n$ the value of the limiting strength, $D_{c}(n)$, is expected to increase, too, both for $n=$ even (for frustrated ladders) and $n=o d d$. In the limit, $n \rightarrow \infty, D_{c}(n)$ very probably tends to infinity, so that the critical behavior of that system is described by a conventional random fixed point. Work is in progress to verify this scenario and to obtain a general physical picture about the low-energ.tproperties of random two-dimensional antiferromagnets 46 .

Acknowledgment: F.I. is grateful to J.-C. Anglès d'Auriac and G. Fáth for useful discussions. This work has been supported by a German-Hungarian exchange 
program (DAAD-MÖB), by the German Research Foundation DFG, by the Hungarian National Research Fund under grant No OTKA TO23642, TO25139, TO34183, MO28418 and M36803, by the Ministry of Education under grant No. FKFP 87/2001 and by the Centre of Excellence ICA1-CT-2000-70029. Numerical calculations are partially performed on the Cray-T3E at Forschungszentrum Jülich.

${ }^{1}$ F.D.M. Haldane, Phys. Lett. 93A, 464 (1983).

${ }^{2}$ I. Affleck, T. Kennedy, E.H. Lieb and H. Tasaki, Phys. Rev. Lett. 59, 799 (1987).

${ }^{3}$ M.P.M. den Nijs and K. Rommelse, Phys. Rev. B 40, 4709 (1989).

${ }^{4}$ For a review see: E. Dagotto and T.M. Rice, Science 271 618 (1996).

${ }^{5}$ E. Dagotto, J. Riera and D.J. Scalapino, Phys. Rev. B 45, 5744 (1992).

${ }^{6}$ E.H. Kim, G. Fáth, J. Sólyom and D.J. Scalapino, Phys. Rev. B 62, 14965 (2000).

${ }^{7}$ M.A. Martin-Delgado, J. Dukelsky and G. Sierra, Phys. Lett. A 250, 431 (1998); N. Flocke, Phys. Rev. B 56, 13673 (1997).

${ }^{8}$ G. Theodorou and M.H. Cohen, Phys. Rev. Lett. 37, 1014 (1976).

${ }^{9}$ M. Hase, K. Uchinokura, R.J. Birgeneau, K. Hirota and G. Shirane, J. Phys. Soc. Jpn. 65, 1392 (1996); M. Hase, N. Koide, K. Manabe, Y. Sasago, K. Uchinokura and A. Sawa, Physica B 215, 164 (1995).

${ }^{10}$ M.C. Martin, M. Hase, K. Hirota and G. Shirane, Phys. Rev. B 56, 3173 (1997).

11 T. Masuda, A. Fujioka, Y. Uchiyama, I. Tsukada, and K. Uchinokura, Phys. Rev. Lett. 80, 4566 (1998).

${ }^{12}$ K. Manabe, H. Ishimoto, N. Koide, Y. Sasago, and K. Uchinokura, Phys. Rev. B 58, R575 (1998).

${ }^{13}$ Y. Ichiyama, Y. Sasago, I. Tsukuda, K. Uchinokura, A. Zheludev, T. Hayashi, N. Miura, and P. Boni, Phys. Rev. Lett. 83, 632 (1999).

${ }^{14}$ D.J. Buttrey, J.D. Sullivan, and A.L. Rheingold, J. Solid. State Chem. 88, 291 (1990).

${ }^{15}$ B. Batlogg, S.W. Cheong, and L.W. Rupp Jr, Physica B 194-196, 173 (1994).

16 J.F. DiTusa, S.W. Cheong, J.H. Park, G. Aeppli, C. Broholm, and C.T. Chen, Phys. Rev. Lett. 73, 1857 (1994).

${ }^{17}$ K. Kojima, A. Keren, L.P. Lee, G.M. Luke, B. Nachumi, W.D. Wu, Y.J. Uemura, K. Kiyono, S. Miyasaka, H. Takagi, and S. Uchida, Phys. Rev. Lett. 74, 3471 (1995).

${ }^{18}$ C. Payen, E. Janod, K. Schoumacker, C.D. Batista, K. Hallberg, and A.A. Aligia, Phys. Rev. B 62, 2998 (2000).

${ }^{19}$ V. Villar, R. Mélin, C. Paulsen, J. Souletie, E. Janod, and C. Payen, cond-mat/0107294.

${ }^{20}$ M. Fabrizio and R. Mélin, Phys. Rev. Lett. 78, 3382 (1997); M. Fabrizio and R. Mélin, Phys. Rev. B 56, 5996 (1997); M. Fabrizio and R. Mélin, J. Phys.:Condens. Matter 9, 10429 (1997). M. Fabrizio, R. Mélin, and J. Souletie, Eur. Phys. J. B 10, 607 (1999); R. Mélin, Eur. Phys. J. B 18, 263
(2000)

${ }^{21}$ R. Mélin, Eur. Phys. J. B 16, 261 (2000);

${ }^{22}$ Y. Uchiyama, Y. Sasago, I. Tsukada, K. Uchinokura, A. Zheludev, T. Hayashi, N. Miura, and P. Böni, Phys. Rev. Lett. 83, 632 (1999).

${ }^{23}$ M. Azuma, M. Takano, R.S. Eccleston, cond-mat/9706170.

${ }^{24}$ T. Miyazaki, M. Troyer, M. Ogata, K. Ueda and D. Yoshioka, J. Phys. Soc. Jpn. 66, 2580 (1997).

${ }^{25}$ S.K. Ma, C. Dasgupta and C.-K. Hu, Phys. Rev. Lett. 43, 1434 (1979); C. Dasgupta and S.K. Ma, Phys. Rev. B22, 1305 (1980).

${ }^{26}$ D.S. Fisher, Phys. Rev. B 50, 3799 (1994).

${ }^{27}$ D.S. Fisher, Phys. Rev. Lett. 69, 534 (1992); Phys. Rev. B 51, 6411 (1995).

28 A.P. Young and H. Rieger, Phys. Rev. B53, 8486 (1996); F. Iglói and H. Rieger, Phys. Rev. Lett. 78, 2473 (1997); A.P. Young, Phys. Rev. B56, 11691 (1997); P. Henelius and S.M. Girvin, Phys. Rev. B 57, 11457 (1998); F. Iglói, R. Juhász and H. Rieger, Phys. Rev. B59, 11308 (1999).

${ }^{29}$ F. Iglói and H. Rieger, Phys. Rev. B57 11404 (1998).

${ }^{30}$ F. Iglói, R. Juhász and H. Rieger, Phys. Rev. B 61, 11552 (2000).

${ }^{31}$ R.A. Hyman and K. Yang, Phys. Rev. Lett. 78, 1783 (1997).

32 C. Monthus, O. Golinelli and Th. Jolicaeur, Phys. Rev. Lett. 79, 3254 (1997).

${ }^{33}$ E. Orignac and T. Giamarchi, Phys. Rev. B 57, 5812 (1997).

34 A.O. Gogolin, A.A. Nersesyan, M. Tsevlik and L. Yu, condmat/9707341.

M.C. Cross and D.S. Fisher, Phys. Rev. B 19, 402 (1979).

36 The notations about "odd" and "even" string topological order are related to the positions of the composite spin-1 operators with an eigenvalue of $S^{z}= \pm 1$, see in Ref. 6 .

${ }^{37}$ F. Iglói, R. Juhász and P. Lajkó, Phys. Rev. Lett. 86, 1343 (2001); F. Iglói, Phys. Rev. B (in print), condmat/0108350

${ }^{38}$ R.B. Griffiths, Phys. Rev. Lett. 23, 17 (1969).

39 B. Boechat, A. Sagnin, and M.A, Continentino, Solid State Commun. 98, 411 (1996).

${ }^{40}$ K. Hida, Phys. Rev. Lett. 83, 3297 (1999); K. Yang and R.A. Hyman, ibid 84, 2044 (2000).

${ }^{41}$ E. Westerberg, A. Furusaki, M. Sigrist and P.A. Lee, Phys. Rev. B55, 12578 (1997).

${ }^{42}$ O. Motrunich, S.-C. Mau, D.A. Huse and D.S. Fisher, Phys. Rev. B61, 1160 (2000).

${ }^{43}$ Y-C. Lin, N. Kawashima, F. Iglói and H. Rieger, Prog. Th. Phys. (Suppl.) 138, 470 (2000); D. Karevski, Y-C. Lin, H. Rieger, N. Kawashima and F. Iglói, Eur. Phys. J. B20, 267 (2001)

44 E. Carlon, P. Lajkó and F. Iglói, Phys. Rev. Lett. (in print), preprint cond-mat/0106325.

45 The strong finite-size dependence of the RG-data indicates that several decimation steps are needed before the asymptotic region of the RG-flow is reached. This type of slow convergence for the zig-zag and the $J_{1}-J_{2}$ ladders is probably connected with the effect of frustration, which is not there for the conventional ladder, with and without dimerization.

${ }^{46}$ R. Mélin et al (unpublished). 